\title{
Partial Malrotation of Bowel Loops in an Adolescent with Left Hypochondrial Pain
}

\author{
Kanwal Laique and Syed Mubarak Ali \\ Department of Diagnostic Radiology, Abbasi Shaheed Hospital, Karachi, Pakistan
}

\begin{abstract}
In the embroyological development, any deviation from physiological midgutrotation results in intestinal malrotation, which may further cause midgutvolulus or intestinal obstruction. It is predominantly a pediatric diagnosis; however, it may occur in adolescent or adult life. A case is presented here of a 17-year boy who came in Accident and Emergency Department, Abbasi Shaheed Hospital with complaint of intermittent and severe intensity pain in left upper quadrant of abdomen. Screening ultrasound abdomen was done, which was unremarkable; then he underwent Doppler evaluation of upper abdomen. Doppler ultrasound depicted abnormal vertical orientation of superior mesenteric vessels. He underwent barium enema, which revealed features of intestinal malrotation. He was further investigated with contrast-enhanced CT abdomen for complications, which confirmed the aforementioned abnormal orientation of superior mesenteric vessels and diagnosis of intestinal malrotation without any complication. In summary, any non-specific abdominal complaint with or without intestinal obstruction in an adolescent or adult patient should be investigated for the possibility of intestinal malrotation.
\end{abstract}

Key Words: Intestinal malrotation, Adolescent, Superior mesenteric artery, Abdominal pain.

How to cite this article: Laique K, Ali SM. Partial malrotation of bowel loops in an adeloscent with left hypochondrial pain. J Coll Physicians Surg Pak 2019; 29 (Supplement 2):S83-S85.

\section{INTRODUCTION}

Midgut malrotation is a medical condition that occurs during embryogenesis due to an abnormal intestinal rotation when it comes back to the abdominal cavity physiologically. The prevalence of intestinal malrotation is $\mathbf{1}$ in $\mathbf{5 0 0}$ live births in the pediatric population. It may present in adolescents and adults. Adult bowel malrotation is very unusual, with an incidence ranging between 0.0001 to $0.19 \% .^{1,2}$ Many individuals can live their entire lives without any symptoms, but the abnormally rotated bowel may turn into acute emergencies like midgut volvulus, internal hernias and intestinal obstruction.

Imaging investigations play a major role in the diagnosis of bowel malrotation. The standard method for diagnosing midgut malrotation is the upper gastrointestinal contrast series which depicts abnormal position of duodenum to the right of spine, small bowel on right and colon on the left side of abdomen. Computational tomography (CT) signs of midgut malrotation include reversed position of super mesenteric artery(SMA) and super mesenteric vein (SMV), the duodenojejunal junction to the right of the midline, the jejunum on the

Correspondence to: Dr. Kanwal Laique, Department of

Diagnostic Radiology, Abbasi Shaheed Hospital, Karachi,

Pakistan

E-mail: kanwallaique@gmail.com

Received: April 30, 2019; Revised: July 05, 2019;

Accepted: July 20, 2019 right and ileal loops on the left side of abdomen. ${ }^{3}$ Normally on CT images, the SMV is positioned on the right side of the SMA.

In this case, the abnormal orientation of SMA and SMV was found on Doppler ultrasound of abdomen and diagnosis was confirmed on future imaging.

\section{CASE REPORT}

A 17-year boy, resident of Orangi Town, Karachi, presented in Emergency Department, Abbasi Shaheed Hospital with a history of intermittent pain in left upper quadrant of abdomen for seven days. Pain was of severe intensity and associated with nausea. No aggravating or relieving factors were identified. There were no recent changes in eating and bowel habits.

Clinical examination revealed unremarkable febrile and hemodynamic status. Abdomen was soft with noteworthy tenderness in the left hypochondrial quadrant and epigastrium.

Hematology and biochemistry were unremarkable. Screening ultrasound of the abdomen was done, which was unremarkable, too. Then he underwent Doppler ultrasound of superior mesenteric vessels. Doppler ultrasound of upper abdomen demonstrated the abnormal anterior location of SMV just in front of the SMA (Figure 1).

He underwent barium enema, which revealed the duodenojejunal junction, jejunal loops on right side and ceacumon the left side of abdomen. He was further investigated with contrast-enhanced CT abdomen for 
complications. It showed failure of duodenojejenal junction to reach across midline towards left, the superior mesenteric vessels vertically oriented as vein being anterior in position to the SMA (Figure 2) and abnormal position of bowel loops, i.e. small intestine on the right and ceacum and large intestine on the left side of abdomen. These findings were consistent with partial midgut malrotation. No complications, like volvulus and ischemic bowel loops, were noted. The patient underwent surgery, Ladd's procedure was carried out, including division of abnormal peritoneal bands, mobilisation of bowel and widening of the mesentery. The recovery was uneventful.

\section{DISCUSSION}

Midgut malrotation occurs in about 1 in 500 newborns and presents in the first month of life ${ }^{4} \mathrm{~A}$ small number of patients may present later in life or may remain symptomless. 5

Partial intestinal abnormal rotation occurs due to failure of embryonic midgut to complete the physiological $270^{\circ}$ rotation during intrauterine gestational period. ${ }^{6}$ It occasionally presents in adults who are symptomless, and diagnosed incidentally. 6 The typical features of midgut malrotation can be identified by sonography, CT or upper gastrointestinal contrast series. ${ }^{7}$ Diagnostic imaging modality of choice in pediatric patients is the upper gastrointestinal contrast study. ${ }^{7}$ Upper gastrointestinal contrast series shows atypical proximal small intestine corkscrew appearance with duodenojejenal junction right to the midline. ${ }^{8}$ In patients of malrotation, a contrast-enhanced CT scan of abdomen typically shows the SMV placed on the left or passes behind the SMA. 4 Colour Doppler ultrasound of the upper abdomen provides a similar finding. ${ }^{9}$ Other CT findings include intestinal obstruction, engorgement of the mesenteric vessels, and abnormal position of small and large bowel. In this reported case, alongwith the typical features of an abnormal position of duodenojejenal junction to the right of spine, small intestinal loops in right and ceacum and large intestine in the left half of abdomen, vertical orientation of superior mesenteric vessels was noted as SMV was anterior in position to the SMA. However, no complications were noticed. This patient underwent surgery, the Ladds's procedure, including division of abnormal peritoneal bands, mobilisation of bowel and widening of mesentry. Prophylactic appendicectomy was also done to avoid mistakes in future for the pain of appendicitis in left lliac fossa, as the normal anatomical positioning is not achieved in this surgical procedure.

Midgut malrotation may lead to potential life-threatening complications. Therefore, patients of adolescent or adult age with any non-specific symptoms of abdominal pain should be examined for abnormal malrotation of bowel loops and subsequently managed with early surgical treatment. 10

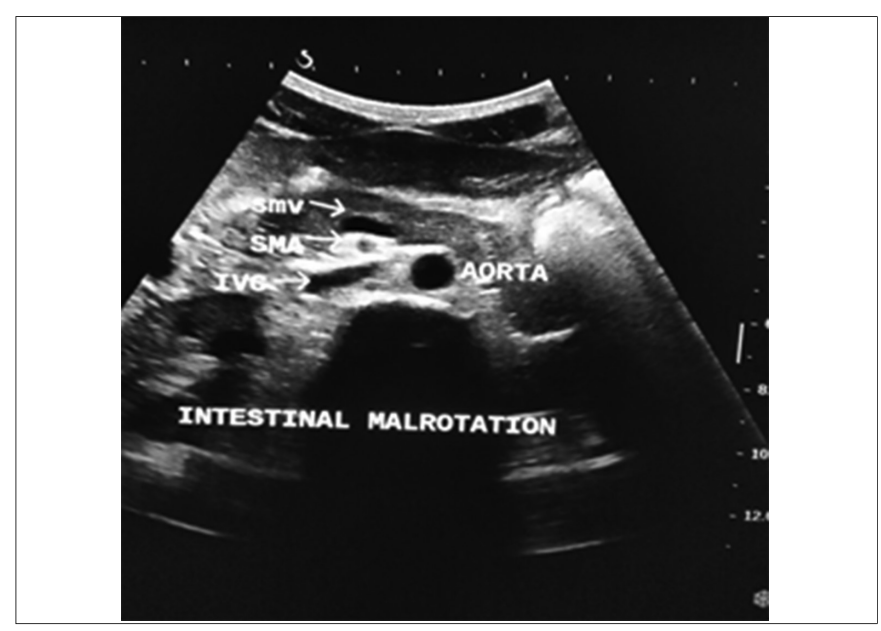

Figure 1: Ultrasound of abdomen showing location of super mesenteric vein in front of superior mesenteric artery.

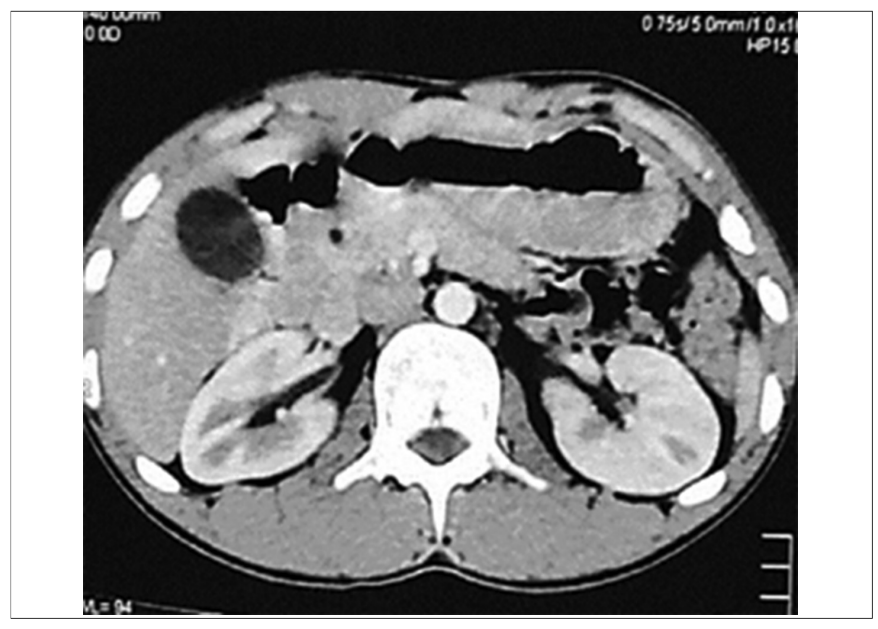

Figure 2: Contrast-enhanced CT scan showing anterior location of superior mesenteric vein.

\section{PATIENT'S CONSENT:}

Informed consent was obtained from the patient to publish the data regarding this case.

\section{CONFLICT OF INTEREST:}

Authors declared no conflict of interest.

\section{AUTHORS' CONTRIBUTION:}

SMA: Supervised to write case report.

$\mathrm{KL}$ : Data collection, literature search, and manuscript writing.

\section{REFERENCES}

1. Liao SF, Yao CC, Chen PR. Whirlpool sign: Typical finding by abdominal computed tomography in a patient with intestinal malrotation and midgut volvulus. Mid Taiwan J Med 2003; 8: 165-8.

2. Guo HY, Feng ST, Li ZP, Wang XY, Sun CH. CT diagnosis of midgut malrotation. Zhonghua Wei Chang Wai Ke Za Zhi 2009; 12:588-90.

3. Dietz DW, Walsh RM, Grundfes-Broniaowski S, Lavery IC, 
Faziot VW, Vog DP. Intestinal malrotation: A rare but important cause of bowel obstruction in adults. Dist Colon Rectum 2002; 45:1381-6.

4. Nichols DM, Li DK. Superior mesenteric vein rotation: A CT sign of midgut malrotation. Amt J Roentgenol 1983; 141:707-8.

5. Ben Ely A, Gorelik N, Cohen-Sivan Y, Zissin R, Carpineta L, Osadchy $A$, et al. Appendicitis in adult with incidental midgut malrotation: CT findings. Clin Radiol 2013; 68:1212-9.

6. Kassir R, Blanc P, Varlet F, Breton C, Lointier P. Gastric bypass with unknown intestinal malrotation: Required attitude. Int $J$ Surg Case Rep 2013; 4:1134-7.

7. Berdon WE. The diagnosis of malrotation and volvulus in the older child and adult: At rap for radiologists. Pediatric Radiol 1995; 25:101-3.

8. Emanuwa OF, Ayantunde AA, DaviestTW. Midgut malrotation first present in gas acute bowel obstructiont in adulthood: A case report and literature review. World J Emerg Surg 2011; 6:22.

9. Pracros JP, Sann L, Genin G, Tran-Minh VA, Morin de Finfe CH, Foray $\mathrm{P}$, et al. Ultrasound diagnosis of midgut volvulus: The 'whirlpool' sign. Pediatr Radiol 1992; 22:18-20.

10. Maxson RT, Franklin PA, Wagner CW. Malrotation in the older child: Surgical management, treatment, and outcome. Am Surg 1995; 61:135-8.

......... 